\title{
Coronary Heart Disease Mortality in Czech Men, 1980-2004
}

\author{
Jindra Reissigová, Marie Tomečková \\ Centre of Biomedical Informatics, Institute of Computer Science AS CR, Prague, Czech Republic
}

Background: The Czech Republic belongs to countries with high coronary heart disease (CHD) mortality. The aim was to analyze age, period and cohort specific CHD mortality in men from the Czech Republic.

Design and Methods: National data on mortality of men (30-74 yrs.) in the Czech Republic in 1980-2004 were explored. The Poisson regression model was applied to evaluate age, period and cohort effects on mortality.

Results: An adequate fit of CHD men's mortality provided the age-period-cohort model $(p=0.121)$. The ratio of mortality of the age group $45-49$ to $40-44$ was by $17 \%$ lower than that of the age group 40-44 to 35-39. The ratio of the relative risk of period $1995-1999$ to $1990-1994$ was by $19 \%$ lower than that of the period 1990-1994 to 1985-1989. The ratios of the relative risks between adjoining birth cohort-groups were close to 1.

Conclusions: The fatal $\mathrm{CHD}$ risk in Czech men was significantly depended on age (the risk increasing with age), calendar period (the risk reduced in the recent years), and birth cohort (the risk decreasing with birth cohort). The crucial positive change in the $\mathrm{CHD}$ mortality trend was observed after the collapse of communism in 1989, and in men past age 40 years.

Keywords: coronary heart disease, cardiovascular, mortality, risk function

\section{Introduction}

At the turn of the $21^{\text {st }}$ century in the European Union countries cardiovascular diseases (CVD) cause between $26 \%$ of deaths (France) and $62 \%$ (Bulgaria) in men and between $31 \%$ of deaths (France) and $71 \%$ (Bulgaria) in women [1]. CVD remain the main cause of death in men and in women in all European countries, except men in France, the Netherlands, and Spain. Over a third of deaths from CVD are from coronary heart disease (CHD), which
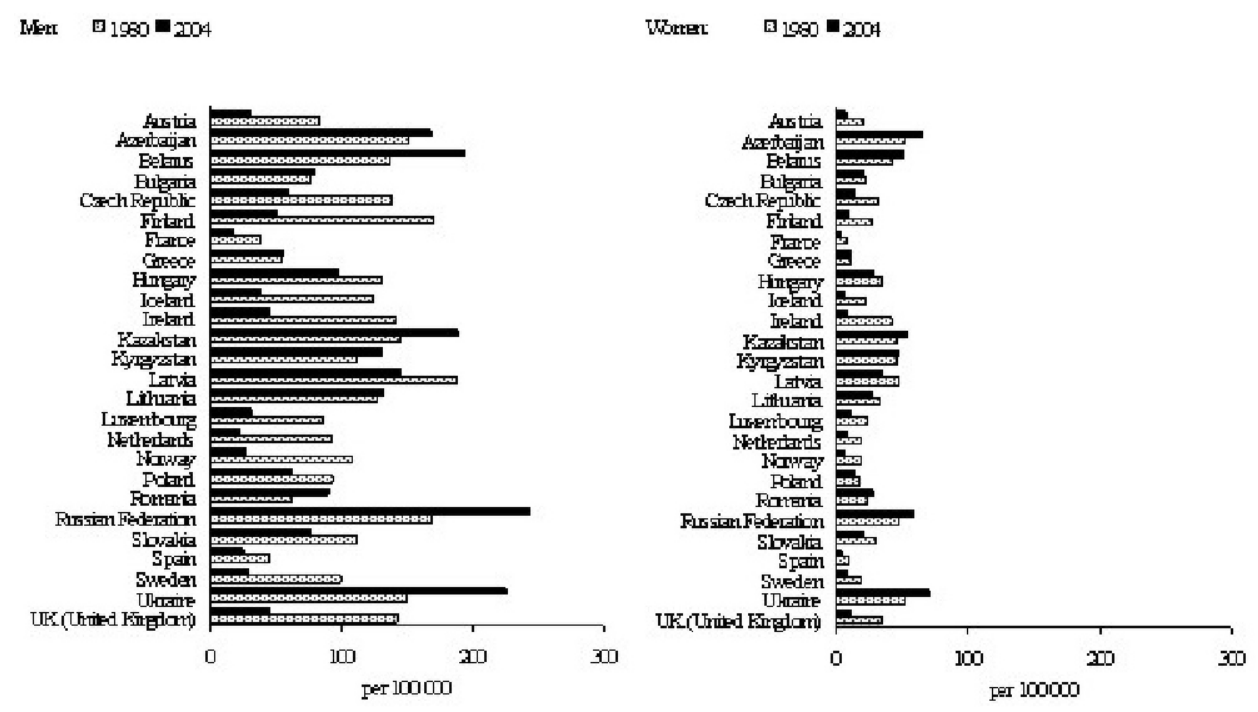

Figure 1. European age-standardized coronary heart disease mortality in some European countries, by sex, 0-64 yrs., 1980', 2004.

${ }^{1}$ In Azerbaijan, Belarus, Kazakstan, Kyrgyzstan, Lithuania and Ukraine mortality in 1981 is presented.Source [1].

by itself is the most common cause of death in Europe. Figure 1 shows agestandardized mortality from CHD in some European countries.

In 1970-2000 mortality from CVD declined almost linearly in western European countries [2]. In eastern European countries, CVD mortality reached a maximum in 1990-1994, followed by a decline in Poland, in Hungary, and in the Baltic states (Estonia, Latvia, Lithuania). An appreciable reversal of the trend was also observed in the Czech Republic (CR) [3]. A main role in the decline in CVD mortality in CR plays most likely a decreasing trend in the occurence of most major CVD risk factors observed in the 1985-2000/01 period [4].

Nevertheless, in 2006 diseases of the circulatory system were the most frequent cause of death in CR (the 2nd most frequent cause of death were malignant neoplasms, the 3rd injuries) [5]. They were responsible for $50.3 \%$ of all deaths. The leading cause of death from the cardiovascular diseases was CHD. It caused $47.1 \%$ of all death from CVD in men, and $40.8 \%$ in women. The aim of this article is to analyze mortality from CHD in Czech men in 1980-2004.

\section{Material and Methods Source data}

The numbers of CHD (codes D410-D414 in the $8^{\text {th }}$ Revision of International Classification of Diseases (ICD-8), codes 410-414 in ICD-9, codes I20-I25 in ICD-10) and mid-year population by the 5 -year age groups $(0-4,5-9, \ldots 80-84,85+)$ in $1980-$ 2004 are from the publications "Population changes in the Czech Republic" yearly issued by the Czech statistical office. Mortality from CHD was directly agestandardized to the European population [6].

\section{Statistical methods}

An age-period-cohort (APC) modelling was applied to analyse mortality from $\mathrm{CHD}$ in men aged 30-74 years from CR in 19802004 (note: mortality in the younger age group was low and the reliability of death cause in the elderly can be arguable, these age groups were excluded from the 
analysis). The number of $\mathrm{CHD}$ deaths was modelled by a log-linear Poisson regression as a function of the age at death, period of death, and birth cohort. The APC modelling was done hierarchically as summarized by Arbyn et al. [7], and finally presented in Table 1 in Results. Agoodness-of-fit of a given model was measured by the residual deviance with a chi-square test. The contribution of an additional term to the model was evaluated by comparing the difference in the deviances between the given model and the model without the term against chisquare distribution with the degree of freedom ( $d f$ ) equalled to the difference in $d f$ between these two models. The adequacy of the model was further verified by the Akaike's information criterion (AIC) for the fit, and an examination of the Pearson residuals. To test for extra-Poisson variation, the Cameron-Trivedi (C-T) test was applied.

It is well known that a linear dependency exists among the variables of age $\left(a_{i}\right.$, $i=1, \ldots, l)$, period $\left(p_{j}, j=1, \ldots, J\right)$ and cohort $\left(c_{k}\right.$, $k=1, \ldots, K): k=l-i+j$. In the model involving the variables of age, period and cohort simultaneously, the linear dependency results in non-unique estimations of these parameters (note: the multiple estimations, however, yield the same fitted number of deaths). To obtain unique estimations, a constraint on the estimated parameters is required. However, the different constraint leads to estimations of age, period and cohort trends. In our case, the constraints of fixing two period parameters (1985-1989, 1990-1994), and one cohort parameter (1935-1944) to zero and the constraints so-called second differences by Clayton and Schifflers [8] were also used to estimate the parameters of the age, period and cohort. Other differences are the contrasts expressed as the ratio (difference on a logarithmic scale) of two adjacent relative values (more in Results). The statistical analysis was performed using the R software version 2.3.1.

\section{Results}

Figure 2 shows the development of agestandardized CHD mortality in men from CR in 1980-2004, in all age groups together, in the age group of 0-64 years, and in the age group of 30-74 years. For

the ability of comparison with mortality in other European countries (Figure 1), this mortality was adjusted (recalculated) on the age-structure of the European standard population.

Figure 3 shows the age, period, and birth cohort specific (yearly) mortality in the age group of 30-74 years. Mortality is plotted on a logarithmic scale. Mortality was increasing with age (Figure 3a), decreasing in the recent years (Figure $3 b$ ), and decreasing with birth cohort (Figure $3 c)$. In other words, Figure 3 depicts mortality data subsequently stratified by age, period and birth cohort, which we have at our disposal for the statistical analysis testing simultaneously to what extent age, period, and birth cohort influence mortality.

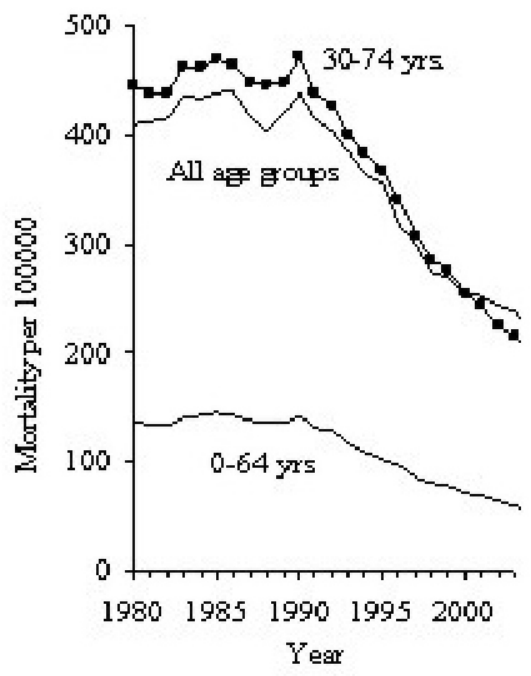

Figure 2. European age-standardized coronary heart mortality in the Czech Republic, men, in all and specific age groups, 1980-2004.

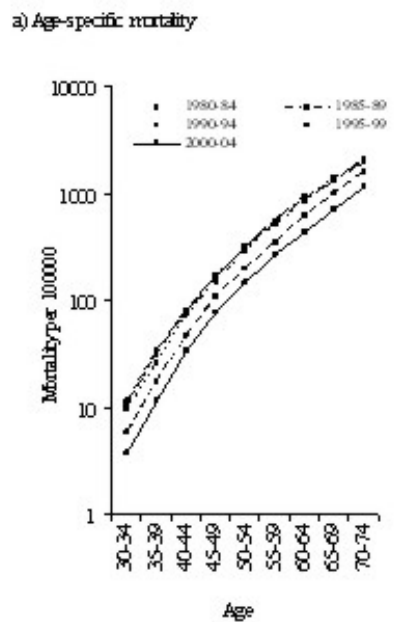

b) Period-specific nutaily

c) Einthcolort-speciri nutality
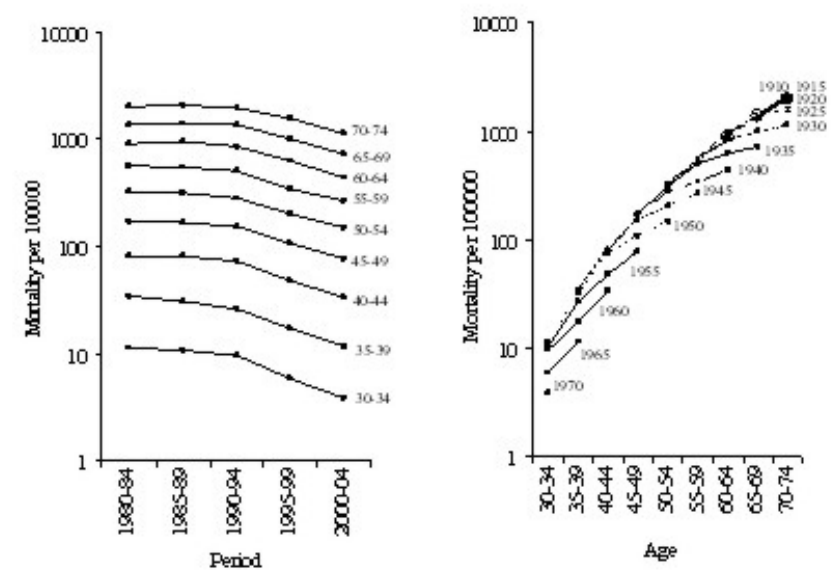

Figure 3. Age, period, birth cohort-specific coronary heart mortality in the Czech Republic, men, 30-74 yrs., 1980-2004'.

${ }^{1}$ In c), birth cohort notation represents the centre of the 10-year interval, e.g. birth cohort of 1940 represents the cohort of men born from 01/01/1935 to 31/12/1944.

The statistical analysis is presented in Table 1, which summaries the results of APC modelling. The effect of age was significant on mortality (the model 1). The fits of the models 2 and $3 a-3 c$ were significantly better compared with the model 1, however, still not sufficient. A more adequate fit provided the APC model (the model 4). It means that the risk of death was significantly depended on all three effects: age, calendar period, and birth cohort. In the APC model, no extraPoisson variation (in other words, no significance difference between mean and variance) was detected $(p=0.500)$, and the
Pearson residuals ranged from -1.864 to 1.784 .

The self effects of age, period, and cohort on mortality are demonstrated in Figure 4. The age effect was expressed as agespecific mortality per 100000 , the period and cohort effects as relative risk of death from $\mathrm{CHD}$. Increasing age was elevating CHD mortality (Figure 4a). The risk of death from CHD was reducing in the most recent periods (Figure 4b, compared with 1985-1995, i.e. the reference period), and with birth cohort (Figure 4c). 
The cohort risks were related to the risk of the cohort 1940 (the reference cohort). The cohort 1940 included men born around (1935-1944) the beginning of the Second World War in 1939. In fact, it was a central cohort and compared with this cohort, the younger and older cohorts are based on fewer data, Figure $3 c$ (it resulted in the varying width of confidence intervals, Figure 4c).

The numbers in brackets in Figure 4 indicate the ratio of two adjacent values. Remind that these ratios are the same for various age, period and cohort parameter estimates, and thus they solve the nonidentifiability problem in the APC model as mentioned in Material and Methods. It is important to emphasize that the ratios indicate neither increasing nor decreasing trend. In fact, the values under 1 indicate "deceleration" of the trend, and the values above 1 indicate "acceleration" of the trend. Let's describe the most essential sudden changes in the trends:

-Following Figure 4a): The ratio of mortality of the age group $45-49$ to the age group $40-44$ was lower by $17 \%$ than that of the age group 40-44 to the age group 35-39, because the mentioned mortality rate was $\left(\frac{165.2}{87.7}\right) /\left(\frac{87.7}{38.6}\right)=83 \%$.

The sequent mortality rates were $89 \%$ for the age group of $45-49$ years, and $93 \%$ $99 \%$ for the age group of 50 years and over. In summary, the largest deceleration of the mortality trend was observed in men in the age group 40-49 years.

-Following Figure $4 b$ ): The ratio of the relative death risk of the period 19951999 to the period 1990-1994 was lower by $19 \%$ than that of the period $1990-1994$ to the period 1985-1989, because the mentioned risk ratio is $\left(\frac{0.81}{1.0}\right) /\left(\frac{1.0}{1.0}\right)=81 \%$.

Other more than $10 \%$ changes in the trend were not observed. So that, the largest decrease in the mortality trend was achieved in 1995-1999 compared to the previous development of the trend.

-Following Figure 4c): No larger sudden changes in the relative death risk were observed by birth cohorts; the ratio of the relative risks between adjoining cohortgroups ranged from 0.92 to 1.06 .

Table 1. Age, period, cohort modelling of coronary heart mortality, men, 30-74 yrs., Czech Republic, 1980-2004.

\begin{tabular}{|c|c|c|c|c|c|c|c|c|c|}
\hline No. ${ }^{1}$ & Model & $\begin{array}{l}\text { Residual } \\
\text { deviance } \\
\text { (D) }\end{array}$ & $\begin{array}{l}\text { Degree of } \\
\text { freedom } \\
\text { (df })\end{array}$ & $p$-value & 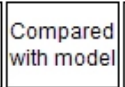 & $\Delta(\mathrm{D})^{2}$ & $\begin{array}{c}\Delta(d f) \\
3\end{array}$ & $p$-value & $\mathrm{AlC}^{4}$ \\
\hline 0 & Interception & 355388.0 & 44 & $<0.001$ & & & & & \\
\hline 1 & Age & 15148.0 & 36 & $<0.001$ & 0 & 340240.0 & 8 & $<0.001$ & 15600.6 \\
\hline 2 & \begin{tabular}{|l} 
Age-Drift \\
\end{tabular} & 3255.5 & 35 & $<0.001$ & 1 & 11892.5 & 1 & $<0.001$ & 3710.1 \\
\hline $3 a$ & Age-Age* Drift & 2922.5 & 27 & $<0.001$ & 2 & 333.0 & 8 & $<0.001$ & 3393.1 \\
\hline $3 \mathrm{~b}$ & Age-Period & 388.2 & 32 & $<0.001$ & 2 & 2867.3 & 3 & $<0.001$ & 848.8 \\
\hline $3 c$ & Age-Cohort & 1872.6 & 24 & $<0.001$ & 2 & 1382.9 & 11 & $<0.001$ & 2349.2 \\
\hline 4 & $\begin{array}{l}\text { Age-Period- } \\
\text { Cohort }\end{array}$ & 28.7 & 21 & 0.121 & $3 b$ & 359.5 & 11 & $<0.001$ & 511.3 \\
\hline
\end{tabular}

${ }^{1}$ Follow Figure 2. If the model 1 holds, the age-specific mortality curves by the periods are identical. If the model 2 applies, the age-specific mortality curves are parallel at constant distances (so-called drift). In the case of model 3a, the age-specific mortality curves are not parallel; the age-specific mortality in successive periods differ by the constant specific for each age group. In the case of the model $3 b$, the age-specific (or equivalently period-specific) mortality curves, are parallel, but at different distances. If the model $3 c$ holds, the birth cohort-specific mortality curves are parallel at different distances. The model 4 allows for the nonparallel age-specific mortality curves as a function of the period and cohort variables. ${ }^{2}$ Difference in residual deviances.

${ }^{3}$ Difference in degree of freedoms. ${ }^{4}$ Akaike's information criterion.
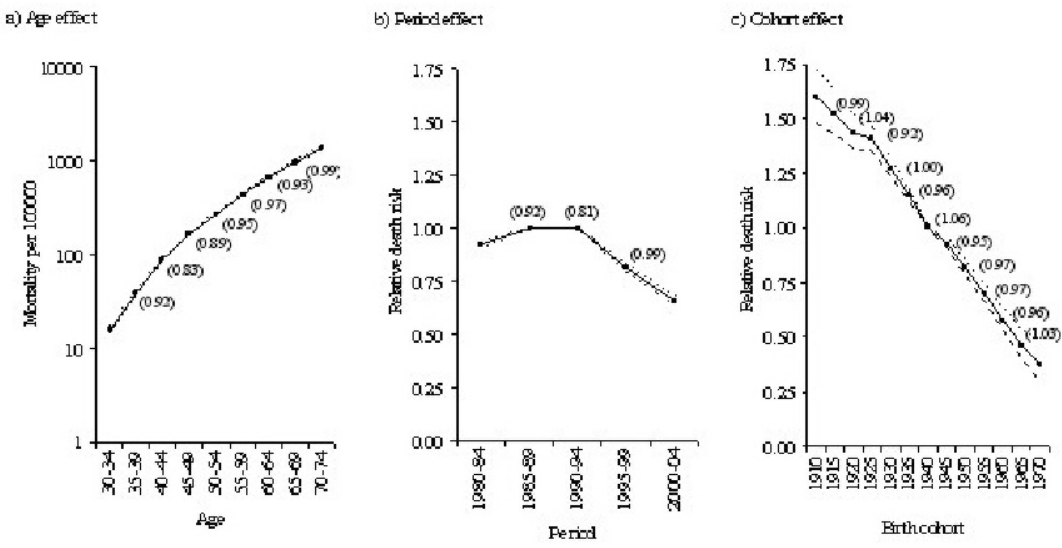

Figure 4. Estimated effects of age, period1 and birth cohort ${ }^{2}$ (with 95\% Cl) on coronary heart mortality in the Czech Republic, men, 30-74 yrs., 1980-2004 (the numbers in brackets indicate the ratio of two adjacent relative risks ${ }^{3}$ ).

${ }^{1}$ Periods of 1985-1989 and 1990-1994 was chosen as reference.

${ }^{2} B$ irth cohort of 1940 was chosen as reference; birth cohort notation represents the centre of the 10year interval, e.g. birth cohort of 1940 represents the cohort of men born from 01/01/1935 to $31 / 12 / 1944$.

${ }^{3}$ For instance, the ratio of mortality of age group 40-44 (87.7) versus age group 35-39 (38.6) to mortality of age group 35-39 (38.6) versus age group 30-34 (15.6) is $(87.7 / 38.6) /(38.6 / 15.6)=0.92$.

\section{Discussion}

APC modelling is used as the method to analyse incidence and mortality from various causes ranged from cancer to cardiovascular diseases. It is a descriptive tool, mainly used for nationally registered data. It helps not only to model but also to predict occurrence of diseases. In this paper, we used APC modelling to explore mortality from $\mathrm{CHD}$ in men from $\mathrm{CR}$.

CVD causes nearly half of all death in Europe (48\%), and CHD by itself is the single most common cause of death in Europe [1]. Primary prevention programmes in many countries attempt to reduce occurrence of $\mathrm{CHD}$ through risk factor modification. However, in studies so far conducted, such interventions have limited the effect on mortality, as summarized in the literature $[9,10]$. On the other hand, as stated there, a small but potentially important benefit (about a 10\% reduction in CHD mortality) may have been missed in those studies. 
Major cardiovascular risk factors are smoking, elevated blood pressure, elevated cholesterol level, and diabetes mellitus. CR belongs to the European countries with high $\mathrm{CHD}$ mortality even if mortality from $\mathrm{CHD}$ has been decreasing in CR from the beginning of 1990 s (Figure 1 ). In this study, using APC modelling, we found out that mortality in Czech men was significantly dependent on the age of death, calendar period of death and birth cohort.

Mortality from $\mathrm{CHD}$ was increasing with age (Figure 4a). The effect of age on mortality was likely to reflect the dependency of mortality on atherosclerosis ("hardening of blood vessels" - the main underlying cause of (VD), which as has been known may begin in childhood, and getting worse as a person gets older. Likely that decisive age for men is the age around 40/50 years, when the largest deceleration of increasing mortality was observed (the mortality ratio of the age group 45-49 to $40-44$ was by $17 \%$ lower than that of the age group 40-44 to 35-39; the mortality ratio of the age group 50-54 to $45-49$ was by $11 \%$ lower than that of the age group 45-49 to 40-44).

The risk of death from $\mathrm{CHD}$ was reduced in the recent period (Figure $4 \mathrm{~b}$ ). The period effect on mortality reflects the risk factors and the level of health care that act in the period of death. The period risks of 19851989, 1990-1994 were fixed to 1 (the reference periods) due to been assumed that the risks were approximately same in these years around the collapse of communism in 1989. Decrease of the risk from fatal $\mathrm{CHD}$ in the recent periods (compared to 1985-1994) is explained by the collapse of communism, followed by lifestyle changes and modern treatment. The ratio of the relative death risks of the period 1995-1999 to the period 1990-1994 was by $19 \%$ lower than that of period 1990-1994 to period 1985-1989.

The relative risk of dying from $\mathrm{CHD}$ was linearly decreasing with birth cohort, and ranged from 0.38 to 1.60 (Figure 4c). No sudden larger trend fluctuations were observed; the ratios of the relative risks between adjoining cohort-groups were close to 1.0. The effect of birth cohort on mortality can be viewed as risk factors and environmental exposures typical for a given generation. Etiology is often related to birth cohort.

One limitation of our APC model is that we did not have data on background characteristics of individuals. Therefore we could not relate the age, period, and cohort trends to trends in cardiovascular risk factors (smoking, blood pressure etc.) and precisely to investigate time from risk factor modification to decline in mortality. Our study is not the analytical study, but the descriptive study, which could help medicine workers understand and speculate about the development of mortality from $\mathrm{CHD}$ in $\mathrm{CR}$. For instance, our data suggests that a sharper decline in mortality was demonstrated in 5-10 years (1995-1999) after the risk factor modification, which is supposed to start around 1990. Furher, even if the aim of our study was not to predict the future CHD mortality trend, mortality among men in CR can be expected to decrease, assuming the trend in the risk factors remain unaltered. The decline is mainly expected due to the fact that the risk of $\mathrm{CHD}$ death is decreasing with the year of birth, and in the recent periods. APC modelling of $\mathrm{CHD}$ was recently also applied in Finland [11] and Japan [12], in which decline in future CHD mortality is also expected. The results of both studies emphasize the significance of maintaining the recent decline of $\mathrm{CHD}$ mortality among middle-aged adults. Few studies on APC mortality were found that were conducted in the past century and predicted development mortality from the past to the beginning of the 21st century. For instance, age-period-cohort effects on CHD mortality in Sweden from 1969 to 1993 were analysed, with forecasts up to 2003 [13]. It would be worth to compare predicted and observed mortality in these studies.

In conclusion, the risk of $\mathrm{CHD}$ death in Czech men was significantly dependent on the age of death, calendar period, and birth cohort. The crucial positive change in the mortality trend was observed after the collapse of communism in 1989, and in men at the age of 40-49 years. Assuming age, period and cohort trends remain unchanged, the further decrease in mortality from $\mathrm{CHD}$ can be expected in Czech men.

\section{References}

[1] European cardiovascular disease statistics 2008 edition. London: British heart foundation, 2008.

[2] Kesteloot H., Sans S., Kromhout D.: Dynamics of cardiovascular and all-cause mortality in Western and Eastern Europe between 1970 and 2000. Eur Heart J 2006; 27(1):107-13.

[3] Levi F., Lucchini F., Negri E., La Vecchia C.: Trends in mortality from cardiovascular and cerebrovascular diseases in Europe and other areas of the world. Heart 2002; 88(2):119-24.

[4] Cifkova R., Skodova Z.: Longitudinal trends in major cardiovascular disease risk factors in the Czech population. Cas Lek Cesk 2004; 143(4):219-226 (in Czech).

[5] Deaths 2006. Institute of Health Information and Statistics of the Czech Republic, Prague 2007. [6] Doll R., Cook P.: Summarizing indices for comparison of cancer incidence data. Int J Cancer, 1967; 2(3):269-279.

[7] Arbyn M., Van Oyen H., Sartor F., Tibaldi F., Molenberghs $\mathrm{G}$.: Description of the influence of age, period and cohort effects on cervical cancer mortality by loglinear Poisson models (Belgium, 1955-94), Arch Public Health 2002; 60:73-100.

[8] Clayton D., Schifflers E.: Models for temporal variation in cancer rates. II: Ageperiod-cohort models. Stat Med 1987; 6(4):469-481.

[9] Ebrahim S.: Systematic review of randomised controlled trials of multiple risk factor intervention for preventing coronary heart disease. BMJ 1997; 314(7095):16661674.

[10] Ebrahim S., Beswick A., Burke M., Davey Smith G.: Multiple risk factor interventions for primary prevention of coronary heart disease. Cochrane Database Syst Rev 2006; (4):CD001561

[11] Huovinen E., Härkänen T., Martelin T., Koskinen S., Aromaa A.: Predicting coronary heart disease mortality-assessing uncertainties in population forecasts and death probabilities by using Bayesian inference. Int J Epidemiol 2006; 35(5):124652.

[12] Ma E., Iso H., Takahashi H., Yamagishi K., Tanigawa T.: Age-Period-Cohort analysis of mortality due to ischemic heart disease in Japan, 1955 to 2000. Circ J 2008; 72(6):966-72. 
[13] Peltonen M., Asplund K.: Age-period-cohort effects on ischaemic heart disease mortality in Sweden from 1969 to 1993, and forecasts up to 2003. Eur Heart 1997; 18(8): 1307-1312.

\section{Acknowledgments}

The paper was supported by the project $1 \mathrm{M} 06014$ of the Ministry of Education, Youth and Sports CR.

\section{Contact}

RNDr. Jindra Reissigová, Ph.D.

$\mathrm{CBI}$, Institute of Computer Science AS CR

Pod Vodárenskou věží 2, Prague 818207

Czech Republic

e-mail: reissigova@euromise.cz 